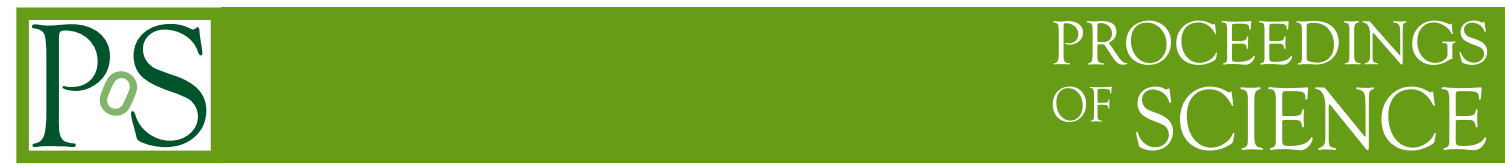

\title{
Searches for Light Higgs Bosons at the CMS Experiment
}

\author{
Alexis Kalogeropoulos * \\ Princeton University \\ E-mail: Alexis.Kalogeropoulosecern.ch
}

We report on recent results by the CMS Collaboration on searches for light Higgs bosons based on a sample of proton-proton collision data at $\sqrt{s}=13 \mathrm{TeV}$, corresponding to a total integrated luminosity of $35.9 \mathrm{fb}^{-1}$.

XXVII International Workshop on Deep-Inelastic Scattering and Related Subjects - DIS2019 8-12 April, 2019

Torino, Italy

*Speaker.

${ }^{\dagger}$ For the CMS Collaboration 


\section{Introduction}

Recent results on the properties of the discovered standard model (SM) like Higgs (H) boson with a mass around $125 \mathrm{GeV}[1,2,3,4,5]$ still support models Beyond the Standard Model (BSM), which advocate the existence of extra Higgs sectors predicting several physical Higgs bosons which can decay into several possible final states mediated by light pseudoscalar bosons (a or $\mathrm{a}_{1}$ ). The analyses presented here have been performed by the CMS Collaboration [6], using the data collected during 2016 and corresponding to a total integrated luminosity of $35.9 \mathrm{fb}^{-1}$.

\section{Search for light bosons decaying to muons $p p \rightarrow h \rightarrow 2 \mathrm{a}+X \rightarrow 4 \mu+X$ (HIG-18-003) [7]}

This is a model independent search for a pair production of new light bosons $\left(h_{1,2}\right)$ decaying to two pairs of muons. It is motivated by next-to-minimal supersymmetric standard model (NMSSM) and supersymmetry (SUSY) models with hidden sectors $[8,9,10]$, resulting in signatures like $p p \rightarrow h \rightarrow 2 \mathrm{a}+X \rightarrow 4 \mu+X$, where $X$ are spectator particles, like dark photons $\left(\gamma_{D}\right)$. The $\gamma_{D}$ can couple to SM particles via $\varepsilon$, which is a small kinetic mixing with SM photons. The lifetime and displacement of the $\gamma_{D}$ depends on its mass and the $\varepsilon$. In the considered models, a SM-like Higgs boson decays via $h \rightarrow 2 n_{1}$, where $n_{1}$ is the lightest non-dark neutralino, which then decays like $n_{1} \rightarrow n_{D}+\gamma_{D}$, with $n_{D}$ been a dark undetected neutralino, while the $\gamma_{D}$ decays to a pair of oppositely charged muons. The analysis probes the mass range between 0.25 and $3.55 \mathrm{GeV}$ for the a and between 0.25 and $8.5 \mathrm{GeV}$ for the $\gamma_{D}$ when $m_{n_{1}}=10 \mathrm{GeV}$ and $m_{n_{D}}=1 \mathrm{GeV}$ respectively.

Events are required to have at least three reconstructed muons, one with $p_{\mathrm{T}}>15 \mathrm{GeV}$ and the other two with $p_{\mathrm{T}}>5 \mathrm{GeV}$, while all must have $|\eta|<2.4$. The main backgrounds are $b \bar{b}, J / \psi$ and electroweak processes with four muons in the final state. The $b \bar{b}$ is estimated from data, while the $J / \psi$ which coming mainly from single and double parton scattering is estimated from a method employing both data and simulation.

With no significant excess over the background only hypotheses, upper limits at $95 \%$ confidence level (CL) are set on the $\sigma\left(p p \rightarrow \mathrm{h}_{1,2} \rightarrow 2 \mathrm{a}_{1}\right) \mathscr{B}^{2}\left(\mathrm{a}_{1} \rightarrow 2 \mu\right)$ for several $m_{\mathrm{h}_{1,2}}$, compared against a predicted rate assuming $\mathscr{B}\left(\mathrm{h}_{1} \rightarrow 2 \mathrm{a}_{1}\right)=0.3 \%, \sigma\left(p p \rightarrow \mathrm{h}_{1}\right)=\sigma_{S M}$ when $\mathrm{m}_{\mathrm{h}_{1}}=125 \mathrm{GeV}$, and $\sigma\left(p p \rightarrow \mathrm{h}_{2}\right) \mathscr{B}\left(\mathrm{h}_{2} \rightarrow 2 \mathrm{a}_{1}\right)=0$ (Fig. 1, left). Further, results at $90 \% \mathrm{CL}$ in interpretations in dark SUSY scenarios, with $\sigma(p p \rightarrow h+X) \mathscr{B}\left(\mathrm{h} \rightarrow 2 \gamma_{\mathrm{D}}+\mathrm{X}\right)$ (Fig. 1, right). These dark SUSY interpretations set new strong constrains on previously unexamined ranges of $\varepsilon$ and $m_{\gamma_{D}}$.

\section{Search for exotic decays of the Higgs boson in a final state with two muons and two b quarks (HIG-18-011) [11]}

This analysis searches for decays of a pair of light pseudoscalars $\mathrm{a}_{1}$, decaying to a pair of opposite sign muons and two b quarks. The gluon and vector boson fusion ( $\mathrm{ggF}$ and VBF respectively) production mechanisms are considered, while as a benchmark a $\mathscr{B}\left(\mathrm{h} \rightarrow \mathrm{a}_{1} \mathrm{a}_{1}\right)=10 \%$ is assumed. The analysis is sensitive to the mass range $20.0<m_{\mathrm{a}_{1}}<62.5 \mathrm{GeV}$, where the upper bounds are imposed by the $\mathrm{H}$ mass. 

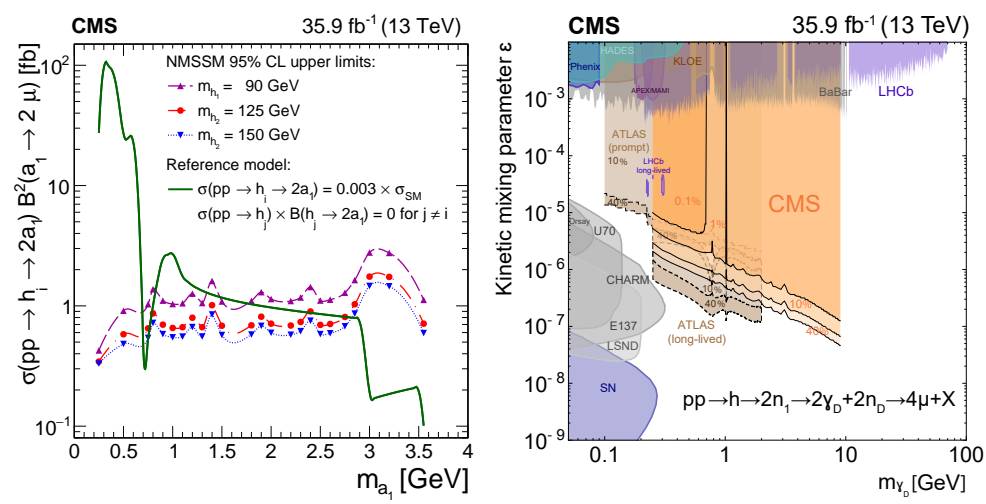

Figure 1: Left: Upper limits at 95\% CL and for different scenarios on the $m_{\mathrm{h}_{1}}$, compared to several reference models as explained in the text. Right: The 90\% CL upper limits interpreted in dark SUSY scenarios in the $\varepsilon, m_{\gamma_{D}}$ plan, overlaid with constraints from other experiments. Plots are taken from Ref. [7].

Two isolated muons with $p_{\mathrm{T}}>20$ and $15 \mathrm{GeV}$ respectively with $|\eta|<2.4$, and 2 b quark jets are required one of which has to pass the tight working points (WP) [14], while the other has to pass either the loose/medium or tight WP, whereas only events with missing transverse momentum $\left(p_{\mathrm{T}}^{\text {miss }}\right)$ less than $60 \mathrm{GeV}$ are considered. The event categorization is based on the $\mathrm{b}$ quark discriminators, and it is basically the different possible combination of the WP, i.e., TightLoose (TL), Tight-Medium (TM) or Tight-Tight (TT). Given that the $b$ quark jets and muons must be the decay products of the $\mathrm{a}_{1}$, it is expected to have $m_{b \bar{b}} \approx m_{\mu \mu} \approx m_{\mathrm{a}_{1}}$, while the reconstructed mass of the muons and the b quarks is expected to be close to $m_{\mathrm{h}}$. A $\chi^{2}$ variable is employed, using the invariant masses of the $b$ quarks and the selected muons, which helps to form the signal regions.

The signal is extracted from an unbinned fit to the $m_{\mu \mu}$ in data simultaneously to all of the TL, TM, TT signal regions. The signal is modelled with a weighted sum of Voigt and Crystal Ball functions, while the background is modelled independently for each category with a set of analytic functions derived by different polynomials as it shown in Fig 2 (left). In the absence of significant excess on the SM background prediction, upper limits on the $\sigma \mathscr{B}\left(\mathrm{h} \rightarrow \mathrm{a}_{1} \mathrm{a}_{1} \rightarrow \mu \mu \mathrm{b} \overline{\mathrm{b}}\right)$ are set at 95\% CL as a function of $m_{\mathrm{a}_{1}}$ which range from 5 to $36 \mathrm{fb}$ and can be seen in Fig. 2 (right). More interpretations on different $2 \mathrm{HDM}+\mathrm{S}$ models can be found in the relevant public result [11].

\section{Search for exotic decays of the Higgs boson in a final state with two muons and two tau leptons (HIG-17-029) [12]}

The analysis probes a masses between 15.0 and $62.5 \mathrm{GeV}$, as due to the boost of the a the analysis has no sensitivity to lower masses. Given the excellent mass resolution of muons, the $\mu \mu+\left(e \mu, e \tau_{h}, \mu \tau_{h}, \tau_{h} \tau_{h}\right)$ final states are considered, which are also favoured in the 2HDM Type III model, while $\tau_{h}$ are isolated tau candidates. The signal is extracted from the dimuon mass spectrum, exploiting the fact the shape is different for different a masses. For the $\mu \mu+e \mu$ and $\mu \mu+\mu \tau_{h}$ final states, a combination of single muon and triple-muon triggers is used, while all of the muons must have $|\eta|<2.4$. Electrons from tau lepton decays are required to have $p_{\mathrm{T}}>7 \mathrm{GeV}$ with $|\eta|<2.5$, while the $\tau_{h}$ candidates must have $p_{\mathrm{T}}>18.5 \mathrm{GeV}$ and $|\eta|<2.3$ 

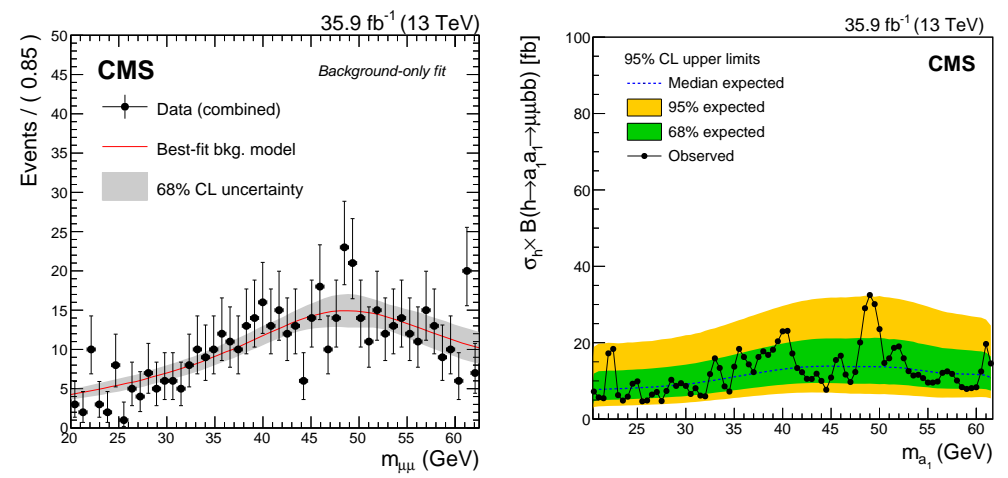

Figure 2: Left: The best fit on data under the background-only hypothesis for all of the TL, TM, TT categories together (left) with 68\% CL uncertainty band for the background model. Right: Observed and expected upper limits at $95 \% \mathrm{CL}$ on the $\sigma \mathscr{B}\left(\mathrm{h} \rightarrow \mathrm{a}_{1} \mathrm{a}_{1} \rightarrow \mu \mu \mathrm{b} \overline{\mathrm{b}}\right)$. Plots are taken from Ref. [11].

Background comes mainly from jets misidentified as leptons which is estimated from data. Such events are mainly coming from $\mathrm{Z}+\mathrm{jets}$, WZ+jets, and the shape is taken from a control region with tau candidates of same-sign and relaxed isolation. The yield is obtained from data with one or two non-isolated tau candidates weighted for the probability for jets to pass the isolation cuts in the signal region. The reconstructed dimuon mass is fitted in order to extract the signal, where in the case of signal simulated events is parametrized with Voigt functions. The parametrizations corresponding to different a masses and for the $2 \mu+\mu \tau_{h}$ final state are shown in Fig 3 (left). No significant excess was found above the SM expectations, and upper limits at 95\% CL are set on the $\left(\sigma_{h} / \sigma_{\mathrm{SM}}\right) \mathscr{B}\left(\mathrm{h}_{1} \rightarrow\right.$ aa $\left.\rightarrow 2 \mu 2 \tau\right)$, which get as low as $1.2 \cdot 10^{-4}$ for a mass of $60 \mathrm{GeV}$ assuming the SM production cross section for the H (Fig. 3, (right)). More interpretations can be found in the relevant public result [12].
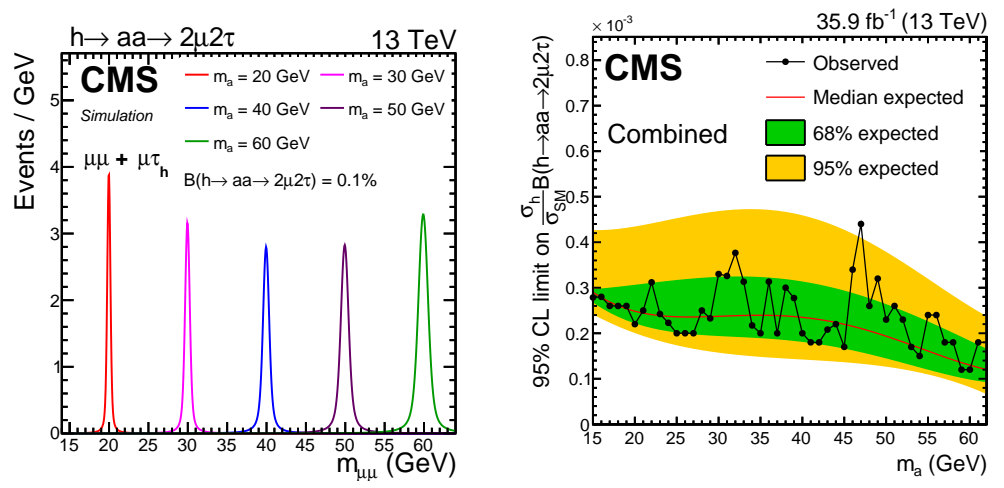

Figure 3: Left: Parameterized dimuon invariant mass of the $\mathrm{h}_{1} \rightarrow \mathrm{aa} \rightarrow 2 \mu 2 \tau$ process for different $m_{\mathrm{a}}$ values in the $2 \mu+\mu \tau_{h}$ final state. Right: Upper limits at $95 \% \mathrm{CL}$ on $\left(\sigma_{h} / \sigma_{\mathrm{SM}}\right) \mathscr{B}\left(\mathrm{h}_{1} \rightarrow\right.$ aa $\left.\rightarrow 2 \mu 2 \tau\right)$. Plots are taken from Ref. [12].

\section{Search for light $\mathrm{a}_{1}$ boson pairs produced in final states with $2 \mu$ and two nearby tracks (HIG-18-006) [13]}

This search probes the mass range from 4 to $15 \mathrm{GeV}$ for the $\mathrm{a}_{1}$, produced from decays of the 
$\mathrm{H}$, considering final states when one of the pseudoscalars decays to a pair of tau leptons, and the other one either into a pair of tau leptons or muons. Although the analysis complements several other light $a_{1}$ searches in different final states, it specifically targets highly Lorentz-boosed muon or tau leptons pairs with overlapping decay products. The analysis focuses primarily on the ggF process, considering same-charge (SC) dimuon events with large angular separation, while each one selected muon is accompanied by one nearby track of opposite charge originating from the same $\mathrm{a}_{1}$ decay and has to be within a cone $\Delta R<0.5$ around the selected muon. The requirement of SC muons, suppresses significantly contributions from top-quark pair, Drell-Yan and diboson production, while QCD multijets make up to $99 \%$ of the total background. Further, the $p_{\mathrm{T}}$ of the leading (subleading) muon has to be higher than 18 (10) GeV, with $|\eta|<2.4$ for both. Several categories of reconstructed tracks are considered which are used to build $\mathrm{a}_{1}$ candidates or to define sideband regions in order to estimate background contributions. Signal is extracted from the $2 \mathrm{D}\left(m_{1}, m_{2}\right)$ distribution of the invariant masses of the the muon-track systems forming the $\mathrm{a}_{1}$ candidates. The $2 \mathrm{D}\left(m_{1}, m_{2}\right)$ is built from a binned template, which takes into account possible correlations between $m_{1}$ and $m_{2}$ and the individual shapes of the corresponding 1D distributions for the two muon-track systems.

The $2 \mathrm{D}\left(m_{1}, m_{2}\right)$ distribution is first unfolded into a one row distribution, which is then fit to extract the signal, as shown in Fig. 4 (left). The results of the analysis are used to set upper limits at 95\% CL on the $\sigma(p p \rightarrow H+X) \mathscr{B}\left(H \rightarrow \mathrm{a}_{1} \mathrm{a}_{1}\right) \mathscr{B}^{2}(\mathrm{a} \rightarrow 2 \tau)$, relative to the $\sigma_{\mathrm{SM}}$ predicted in the SM and are shown in Fig. 4 (right). The new limits significantly improve the previous results between $30 \%$ (for low $\mathrm{a}_{1}$ masses) to $80 \%$ (for intermediate masses of $8 \mathrm{GeV}$ ), while it further extends the coverage of $m_{\mathrm{a}_{1}}$ up to $15 \mathrm{GeV}$.
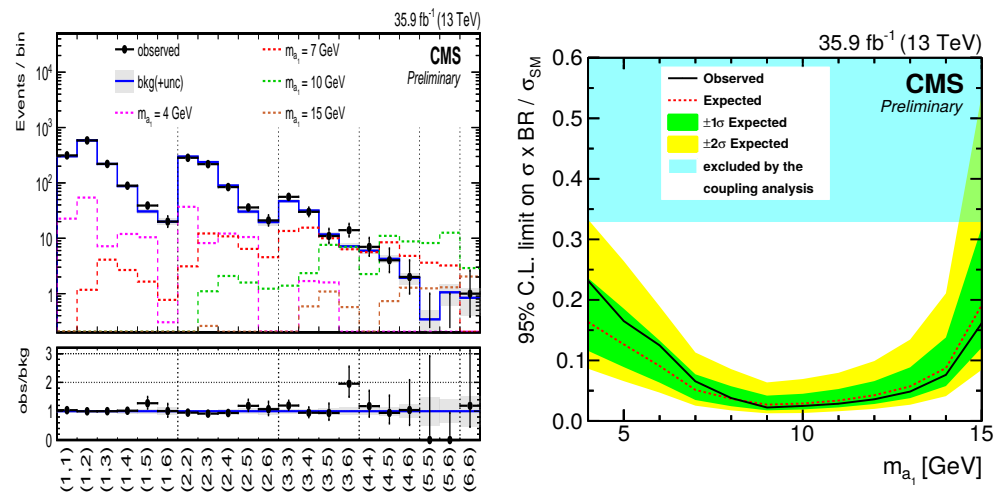

Figure 4: Left: The $\left(m_{1}, m_{2}\right)$ in one row distribution used to extract the signal. Several signal masses are also shown. The signal normalization assumes that the $\mathrm{H}$ is produced with the rate predicted by the SM, defcaying to the $a_{1} a_{1} \rightarrow \tau$ final state with a branching fraction of $20 \%$. The lower plot shows the ratio of the observed data events to the expected background yield. Right: The observed and expected upper 95\% CL limits on the $\sigma(p p \rightarrow H+X) \mathscr{B}\left(H \rightarrow \mathrm{a}_{1} \mathrm{a}_{1}\right) \mathscr{B}^{2}(\mathrm{a} \rightarrow 2 \tau)$, relative to the $\mathrm{H}$ production cross section as predicted by the SM. The shaded area indicates the excluded value of the branching fraction of the $\mathrm{H}$ decay into non-SM particles at 95\% CL from Ref. [2]. Plots are taken from Ref. [13]. 


\section{Summary}

Recent results by the CMS Collaboration on searches for light Higgs bosons with extended scalar sectors have been presented using proton-proton collision data at $\sqrt{s}=13 \mathrm{TeV}$, corresponding to a total integrated luminosity of $35.9 \mathrm{fb}^{-1}$ recorded by the CMS experiment at the LHC. Several different mass ranges are probed for different final states. No excess of data over the background predictions has been observed in any of the performed analyses, and upper limits at 95\% confidence level on the product of the cross section and branching fraction for several signal processes are set. The full details of the analyses can be found in the relevant public results.

\section{References}

[1] G. Aad et al. [ATLAS Collaboration], Phys. Lett. B 716 (2012) 1 doi:10.1016/j.physletb.2012.08.020 [arXiv:1207.7214 [hep-ex]].

[2] G. Aad et al. [ATLAS and CMS Collaborations], JHEP 1608, 045 (2016) doi:10.1007/JHEP08(2016)045 [arXiv:1606.02266 [hep-ex]].

[3] A. M. Sirunyan et al. [CMS Collaboration], Eur. Phys. J. C 79 (2019) no.5, 421 doi:10.1140/epjc/s10052-019-6909-y [arXiv:1809.10733 [hep-ex]].

[4] S. Chatrchyan et al. [CMS Collaboration], JHEP 1306 (2013) 081 doi:10.1007/JHEP06(2013)081 [arXiv:1303.4571 [hep-ex]].

[5] S. Chatrchyan et al. [CMS Collaboration], Phys. Lett. B 716 (2012) 30 doi:10.1016/j.physletb.2012.08.021 [arXiv:1207.7235 [hep-ex]].

[6] S. Chatrchyan et al. [CMS Collaboration], JINST 3 (2008) S08004. doi:10.1088/1748-0221/3/08/S08004

[7] A. M. Sirunyan et al. [CMS Collaboration], [arXiv:1812.00380 [hep-ex]].

[8] S. P. Martin Adv. Ser. Direct. High Energy Phys. 21 (2010) 1 [Adv. Ser. Direct. High Energy Phys. 18 (1998) 1] doi:10.1142/9789812839657_0001, 10.1142/9789814307505_0001 [hep-ph/9709356].

[9] N. Arkani-Hamed, D. P. Finkbeiner, T. R. Slatyer and N. Weiner, Phys. Rev. D 79, 015014 (2009) doi:10.1103/PhysRevD.79.015014 [arXiv:0810.0713 [hep-ph]].

[10] M. Baumgart, C. Cheung, J. T. Ruderman, L. T. Wang and I. Yavin, JHEP 0904, 014 (2009) doi:10.1088/1126-6708/2009/04/014 [arXiv:0901.0283 [hep-ph]].

[11] A. M. Sirunyan et al. [CMS Collaboration], Phys. Lett. B 795, 398 (2019) doi:10.1016/j.physletb.2019.06.021 [arXiv:1812.06359 [hep-ex]].

[12] A. M. Sirunyan et al. [CMS Collaboration], JHEP 1811, 018 (2018) doi:10.1007/JHEP11(2018)018 [arXiv:1805.04865 [hep-ex]].

[13] A. M. Sirunyan et al. [CMS Collaboration], CMS-PAS-HIG-18-006 https://cds.cern.ch/record/2667402

[14] A. M. Sirunyan et al. [CMS Collaboration], JINST 13 (2018) no.05, P05011 doi:10.1088/1748-0221/13/05/P05011 [arXiv:1712.07158 [physics.ins-det]].

[15] A. M. Sirunyan et al. [CMS Collaboration], JHEP 1711, 047 (2017) doi:10.1007/JHEP11(2017)047 [arXiv:1706.09936 [hep-ex]].

[16] G. Aad et al. [ATLAS Collaboration], Phys. Rev. D 90, no. 5, 052004 (2014) doi:10.1103/PhysRevD.90.052004 [arXiv:1406.3827 [hep-ex]]. 